medRxiv preprint doi: https://doi.org/10.1101/2021.10.20.21265149; this version posted October 22, 2021. The copyright holder for this preprint (which was not certified by peer review) is the author/funder, who has granted medRxiv a license to display the preprint in perpetuity.

All rights reserved. No reuse allowed without permission.

\title{
Differences in COVID-19 Risk by Race and County-Level Social Determinants of Health Among Veterans
}

Keywords: Veterans, COVID-19, Social Determinants of Health, County-Level, Race, Health Disparities

Hoda S. Abdel Magid, PhD ${ }^{1-3 *}$, Jacqueline M. Ferguson, $\mathrm{PhD}^{1,4 *}$ Raymond V. Cleve, $\mathrm{PhD}^{1}$, Amanda L. Purnell, $\mathrm{PhD}^{5}$, Thomas F. Osborne, MD ${ }^{1,6}$

${ }^{*}$ Authors contributed equally

1. VA Palo Alto Healthcare System, US Department of Veterans Affairs, Palo Alto, CA, USA

2. Department of Epidemiology and Population Health, Stanford University, Stanford, CA, USA

3. Public Health Program, Santa Clara University, Santa Clara, CA, USA

4. Stanford Center for Population Health Sciences, Stanford University School of Medicine, Stanford, CA, USA

5. Veterans Affairs Central Office, Washington DC, USA

6. Department of Radiology, Stanford University, Stanford, CA, USA

\section{Corresponding Author:}

Hoda S. Abdel Magid, MHS, PhD

Center for Innovation to Implementation

Veterans Affairs Palo Alto Health Care System

MDP-140

795 Willow Road

Menlo Park, California, 94025

United States

Hoda.AbdelMagid@VA.gov

Hoda S. Abdel Magid, MHS, PhD

Postdoctoral Scholar

Department of Epidemiology and Popualtion Health

Stanford University

258 Campus Drive

HRP Redwood Building, Room T258

Stanford, California, 94305

United States

hmagid@stanford.edu

Tables: 3

Figures: 0

Word Count: 1, 222

Abstract Word Count: 100 
medRxiv preprint doi: https://doi.org/10.1101/2021.10.20.21265149; this version posted October 22, 2021. The copyright holder for this preprint (which was not certified by peer review) is the author/funder, who has granted medRxiv a license to display the preprint in perpetuity.

All rights reserved. No reuse allowed without permission.

\section{Abstract}

\section{0 words}

COVID-19 disparities by area-level social determinants of health (SDH) may be impacting U.S. Veterans. This retrospective analysis utilized COVID-19 data from the U.S. Department of Veterans Affairs (VA)'s EHR and geographically linked county-level data from 18 area-based socioeconomic measures. The risk of testing positive with Veterans' county-level SDHs adjusting for demographics, comorbidities, and facility characteristics was calculated using generalized linear models. We found an exposure-response relationship whereby individual COVID-19 infection risk increased with each increasing quartile of adverse county-level SDH such as the percentage of residents in a county without a college degree, eligible for Medicaid, and living in crowded housing. 
medRxiv preprint doi: https://doi.org/10.1101/2021.10.20.21265149; this version posted October 22, 2021. The copyright holder for this preprint (which was not certified by peer review) is the author/funder, who has granted medRxiv a license to display the preprint in perpetuity.

All rights reserved. No reuse allowed without permission.

\section{$\underline{\text { Introduction }}$}

Disparities in COVID-19 infection and mortality vary across the US. ${ }^{1-3}$ These disparities, particularly among racial and ethnic minorities, may be driven by area-level social determinants of health $(\mathrm{SDH})$ and structural resources. ${ }^{4-6}$ In this report, we combine electronic health record (EHR) data from the U.S. Department of Veterans Affairs (VA) with county-level characteristics to assess associations between area-level SDH and COVID-19 infection risk among Veterans with the goal of optimizing care and prevention strategies for our patients.

\section{Methods}

We retrospectively examined records from Veterans actively enrolled in VA healthcare and who were tested for SARS-CoV-2 at VA between February 8, 2020 and December 28, 2020. Methods have been previously described in detail. ${ }^{7}$ In brief, we included demographic characteristics from the VA's EHR database and used the Veteran's home zip code to geographically link publicly available area-based SDH as it has been previously identified being critical for COVID-19 health equity in previous literature. ${ }^{1,8-12}$ A detailed table describing each county-level $\mathrm{SDH}$, source, and original variable name from the source are provided in Supplementary Table 1. We categorized each area-based SDH into quartiles according to the positive case distribution in our analytic sample. We excluded Veterans missing county-level SDH and one VHA facility with fewer than 5 COVID-19 positive cases. The final analytic sample comprised 778,599 Veterans.

\section{Statistical Analysis}

We used generalized linear models to report risk ratios and $95 \%$ confidence intervals for the risk of testing COVID-19 positive for key SDH. To examine effect modification by race between SDH and COVID-19 positivity risk, we stratified our analysis by race including White, Black, and Other Veterans (includes Asian, American Indian/Alaska Native, Native Hawaiian/Other Pacific Islander). All models were adjusted for individual demographics, facility 
medRxiv preprint doi: https://doi.org/10.1101/2021.10.20.21265149; this version posted October 22, 2021. The copyright holder for this preprint (which was not certified by peer review) is the author/funder, who has granted medRxiv a license to display the preprint in perpetuity.

All rights reserved. No reuse allowed without permission.

characteristics, state, and other SDH characteristics that are important for health equity but not identified a-priori as primary SDH characteristics of interest. ${ }^{1,8-12}$ Model standard errors are clustered by VA facility. We conducted all statistical analyses using Stata Version 15 (StataCorp LLC). This quality assessment project received a Determination of Non-Research from Stanford Institutional Review Board as well as by VA determination.

\section{$\underline{\text { Results }}$}

As of December 28, 2020, among the 779,599 Veterans tested at VA, 77,692 (10\%) tested positive for COVID-19. Compared with White Veterans, Black and Other Veterans on average lived in counties with higher percentages of non-US born residents, with higher percentage of non-White residents, individuals without a high school diploma, persons receiving food stamps/SNAP benefits; persons living in crowded housing, persons without broadband, persons living in multigenerational housing (i.e., households where grandparents have children who are under 18); and persons in deep poverty.

We found an exposure-response relationship with individual infection risk of COVID-19 increasing with each increasing quartile of adverse county-level SDH for the following SDH: percentage of residents in a county without a college degree, percentage eligible for Medicaid, and the percentage of residents living in crowded housing. (Table 2). The risk of testing positive for COVID-19 among Veterans living in counties with the top quartile of percentage of residents without a college degree compared to Veterans living in counties in the bottom quartile was 1.23 [95\% confidence interval $(\mathrm{Cl}): 1.10,1.37]$. Veterans living in the top quartile of counties with Medicaid eligibility were 1.17 [95\% Cl: $1.05,1.37]$ times more likely to test positive for COVID19 compared to Veterans living in the bottom quartile. Additionally, the relative risk of testing positive for COVID-19 among all Veterans living in the third quartile of crowded housing was $1.10[95 \% \mathrm{Cl}: 1.04,1.17]$ compared to the first quartile of persons in crowded housing. The association between county-level SDH and COVID-19 cases varied in race-stratified models. The relative risk for testing positive for COVID-19 among Black Veterans living in counties in the 
medRxiv preprint doi: https://doi.org/10.1101/2021.10.20.21265149; this version posted October 22, 2021. The copyright holder for this preprint (which was not certified by peer review) is the author/funder, who has granted medRxiv a license to display the preprint in perpetuity.

All rights reserved. No reuse allowed without permission.

top versus bottom quartile of percentage of persons who are non-White was 1.16 (95\% Cl: 1.01 , 1.33), however, among White Veterans the RR was attenuated $(1.08(95 \% \mathrm{Cl}: 0.95,1.10))$. Among Black Veterans living in counties in the top versus bottom quartile of percentage of households with multigenerational housing, the risk of testing positive for COVID-19 was 1.14 (95\% Cl: $1.04,1.25)$, yet among White Veterans the RR was 1.01 (95\% Cl: 0.93, 1.10). Among Other Veterans, living in a county in the top versus bottom quartile of percentage of residents 25 years or older without $4+$ years of college education was associated with a $31 \%(95 \% \mathrm{Cl}: 1.09$ 1.59) higher risk of testing positive for COVID-19 versus the lowest quartile. Comparing the top versus the bottom quartile, little to no differences were seen among the percentage of persons in deep poverty, percentage without a computer or broadband, and percentage non-US-born residents.

\section{Discussion}

Our results show that Veterans living in areas with lower education levels, higher Medicaid eligibility, crowded housing, non-White residents, and multigenerational housing are experiencing higher risks of COVID-19 infection, a trend which has been noted in other evaluations. ${ }^{1,3,10,13}$ Notably our assessment revealed important associations for our Veterans, such as percentage of residents who are non-White, multigenerational housing, and percentage of residents without a college degree varied in race-stratified models, strengthening for Black and Other Veterans, compared to White Veterans which provides important insights for our targeted interventions.

A strength of our work is that our findings also demonstrate the association between distinct county-level SDH and COVID-19 cases which was possible due to the large cohort size from a nationwide database from the largest integrated healthcare system in the United States. Moreover, our assessment was designed to provide a more precise evaluation to direct targeted enhancement for of our patients which was also achieved by reducing confounding factors from 
medRxiv preprint doi: https://doi.org/10.1101/2021.10.20.21265149; this version posted October 22, 2021. The copyright holder for this preprint (which was not certified by peer review) is the author/funder, who has granted medRxiv a license to display the preprint in perpetuity.

All rights reserved. No reuse allowed without permission.

chronic health conditions which are more common in our population and attenuate the effects of individual-level socioeconomic and VA facility-level characteristics.

\section{Limitations}

Our evaluation is focused on evaluating the association of area-level county-level SDH and COVID-19 test and test positivity of our unique Veteran population, who are on average are male, older, and have more comorbidities than the general US population, which limits generalizability. ${ }^{13}$ Furthermore, our evaluation does not assign weights to the county-level SDH relative to each other since there is no strong evidence to rigorously assign importance across categories. ${ }^{1}$ The association between COVID-19 infection risk and Veterans' county-level SDH may be stronger than the estimated results presented here owing to the fact that some of the covariates adjusted for in this analysis may likely be mediators in the pathway, which would attenuate risk. Lastly, Veterans' home address may not fully capture where Veterans spend most of their time which may result in exposure misclassification, however, we anticipate misclassification would be attenuated by county-level aggregation.

\section{Conclusion}

In this evaluation of Veterans, we identified that county-level SDH factors influence COVID-19 infection risk, informing our understanding of how to improve care strategies, targeted interventions, policy, and resource allocation for Veterans. 


\section{References:}

1. Chin T, Kahn R, Li R, et al. US-county level variation in intersecting individual, household and community characteristics relevant to COVID-19 and planning an equitable response: a cross-sectional analysis. BMJ open. 2020;10(9):e039886.

2. Rentsch CT, Kidwai-Khan F, Tate JP, et al. Patterns of COVID-19 testing and mortality by race and ethnicity among United States veterans: A nationwide cohort study. PLoS medicine. 2020;17(9):e1003379.

3. Chen JT, Krieger N. Revealing the Unequal Burden of COVID-19 by Income, Race/Ethnicity, and Household Crowding: US County Versus Zip Code Analyses. Journal of Public Health Management and Practice. 2021;27:S43-S56.

4. Webb Hooper M, Nápoles AM, Pérez-Stable EJ. COVID-19 and Racial/Ethnic Disparities. JAMA. 2020;323(24):2466-2467.

5. Yancy CW. COVID-19 and african americans. Jama. 2020;323(19):1891-1892.

6. Bassett MT, Chen JT, Krieger N. Variation in racial/ethnic disparities in COVID-19 mortality by age in the United States: A cross-sectional study. PLoS medicine. 2020;17(10):e1003402.

7. Ferguson JM, Abdel Magid HS, Purnell AL, Kiang MV, Osborne TF. Differences in COVID-19 Testing and Test Positivity Among Veterans, United States, 2020. Public Health Reports.0(0):00333549211009498.

8. Raifman MA, Raifman JR. Disparities in the population at risk of severe illness from COVID-19 by race/ethnicity and income. American journal of preventive medicine. 2020;59(1):137-139.

9. Miller IF, Becker AD, Grenfell BT, Metcalf CJE. Disease and healthcare burden of COVID-19 in the United States. Nature Medicine. 2020;26(8):1212-1217.

10. Wrigley-Field E. US racial inequality may be as deadly as COVID-19. Proceedings of the National Academy of Sciences. 2020;117(36):21854-21856.

11. Tan AX, Hinman JA, Abdel Magid HS, Nelson LM, Odden MC. Association Between Income Inequality and County-Level COVID-19 Cases and Deaths in the US. JAMA Network Open. 2021;4(5):e218799-e218799.

12. Nagata JM, Ganson KT, Whittle HJ, et al. Food Insufficiency and Mental Health in the U.S. During the COVID-19 Pandemic. Am J Prev Med. 2021;60(4):453-461.

13. Wang J, Cidade M, Larsen M, et al. 2018 survey of veteran enrollees' health and use of health care. In: Department of Veterans Affairs Rockville, MD; 2019. 
Table 1. Individual and County-level Demographic and Social Determinants of Health Characteristics among U.S. Veterans enrolled in active care in the Veterans Health Administration (VHA), February 8 - December 28, 2020.

\begin{tabular}{|c|c|c|c|c|c|}
\hline N (\%) & $\begin{array}{c}\text { All } \\
(\mathrm{N}=778,599)\end{array}$ & $\begin{array}{c}\text { White } \\
(\mathrm{N}=526,480)\end{array}$ & $\begin{array}{c}\text { Black } \\
\mathrm{N}=186,373\end{array}$ & $\begin{array}{c}\text { Other } \\
(\mathrm{N}=65,746) \\
\end{array}$ & 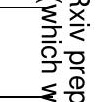 \\
\hline \multicolumn{6}{|l|}{ SARS-CoV-2 } \\
\hline Negative & 700,907 (90.0) & $476,642(90.5)$ & $165,708(88.9)$ & $58,557(89.1)$ & 우 \\
\hline Positive & $77,692(10.0)$ & $49,838(9.5)$ & $20,665(11.1)$ & $7,189(10.9)$ & \\
\hline \multicolumn{6}{|l|}{ Sex } \\
\hline Male & $691,365(88.8)$ & $477,006(90.6)$ & $157,087(84.3)$ & $57,272(87.1)$ & \\
\hline Female & $87,234(11.2)$ & $49,474(9.4)$ & $29,286(15.7)$ & $8,474(12.9)$ & \\
\hline \multicolumn{5}{|l|}{ Age } & \\
\hline $18-34$ & $52,308(6.7)$ & $34,901(6.6)$ & $10,309(5.5)$ & $7,098(10.8)$ & \\
\hline $35-44$ & $78,661(10.1)$ & $51,726(9.8)$ & $17,560(9.4)$ & $9,375(14.3)$ & \\
\hline $45-54$ & $97,506(12.5)$ & $59,319(11.3)$ & $28,597(15.3)$ & $9,590(14.6)$ & \\
\hline $55-64$ & $164,301(21.1)$ & $96,631(18.4)$ & $54,890(29.5)$ & $12,780(19.4)$ & \\
\hline $65-74$ & $248,054(31.9)$ & $177,486(33.7)$ & $53,397(28.7)$ & $17,171(26.1)$ & \\
\hline $75+$ & $137,769(17.7)$ & $106,417(20.2)$ & $21,620(11.6)$ & $9,732(14.8)$ & \\
\hline \multicolumn{5}{|l|}{ Race } & \\
\hline White & $526,480(67.6)$ & -- & -- & -- & \\
\hline Black/African American & $186,373(23.9)$ & -- & -- & -- & \\
\hline Asian & $9,665(1.2)$ & -- & -- & $9,665(14.7)$ & \\
\hline American Indian/Alaska Native & $7,485(1.0)$ & -- & -- & $7,485(11.4)$ & \\
\hline Pacific Islander/Native Hawaiian & $6,874(0.9)$ & -- & -- & $6,874(10.5)$ & \\
\hline Unknown/Missing & $41,722(5.4)$ & -- & -- & $41,722(63.5)$ & \\
\hline \multicolumn{5}{|l|}{ Ethnicity } & \\
\hline Hispanic or Latino & $57,801(7.4)$ & $40,944(7.8)$ & $3,329(1.8)$ & $13,528(20.6)$ & \\
\hline Not Hispanic or Latino & $703,052(90.3)$ & $480,900(91.3)$ & $181,434(97.3)$ & $40,718(61.9)$ & \\
\hline Unknown & $17,746(2.3)$ & $4,636(0.9)$ & $1,610(0.9)$ & $11,500(17.5)$ & \\
\hline \multicolumn{5}{|l|}{ Marital Status } & \\
\hline Single & $130,442(16.8)$ & $76,932(14.6)$ & $40,493(21.7)$ & $13,017(19.8)$ & \\
\hline Married & $365,781(47.0)$ & $264,479(50.2)$ & $69,966(37.5)$ & $31,336(47.7)$ & \\
\hline Divorced/Separated/Widowed & $273,214(35.1)$ & $180,150(34.2)$ & $74,048(39.7)$ & $19,016(28.9)$ & \\
\hline \multicolumn{5}{|l|}{ Urban/Rural } & \\
\hline Urban & $573,072(73.6)$ & $355,834(67.6)$ & $164,582(88.3)$ & $52,656(80.1)$ & \\
\hline Rural & $205,527(26.4)$ & $170,646(32.4)$ & 21,791 (11.7) & $13,090(19.9)$ & \\
\hline \multicolumn{5}{|l|}{ Region } & \\
\hline Continental & $114,974(14.8)$ & $75,113(14.3)$ & $30,589(16.4)$ & $9,272(14.1)$ & \\
\hline Midwest & $160,422(20.6)$ & $121,541(23.1)$ & $29,694(15.9)$ & $9,187(14.0)$ & \\
\hline Northeast & $184,049(23.6)$ & $119,302(22.7)$ & $54,442(29.2)$ & $10,305(15.7)$ & \\
\hline Pacific & $155,350(20.0)$ & $105,629(20.1)$ & $22,431(12.0)$ & $27,290(41.5)$ & \\
\hline Southeast & $163,804(21.0)$ & $104,895(19.9)$ & $49,217(26.4)$ & $9,692(14.7)$ & \\
\hline
\end{tabular}


No Service Disability

Low Income

Low/Moderate Disability High Disability
89,103 (11.4)

154,047 (19.8)

151,646 (19.5)

383,803 (49.3)
62,920 (12.0)

105,635 (20.1)

$108,431(20.6)$

249,494 (47.4)
$18,647(10.0)$

37,995 (20.4)

30,684 (16.5)

99,047 (53.1)
7,536 (11.5)

10,417 (15.8)

12,531 (19.1)

35,262 (53.6)
Median (P25- P75)
Percentage without High School Diploma,

Ages 25+

Percentage without 4+ Years College, Ages

25+

Percentage Food Stamps/SNAP Recipients

Percentage without Health Insurance, Under Age 65

Percentage Eligible for Medicaid, All Ages, 2012

Percentage in Crowded Housing

Percentage 65+ living alone

Percentage of Households without a computer

Percentage of households without broadband

Percentage Non-US-born residents

Percentage Non-White

Median Household Income (thousands)

Unemployment Rate Ages 16+, 2017

Percentage of households where

grandparent have children under 18

Percentage Persons 65+ in Deep Poverty

Percentage of Persons in Deep Poverty

prity-based enrollment system enacte

All
$(\mathbf{N}=778,599)$

$69.9(65.1-77.8)$

$14.6(10.8-18.3)$

$9.7(6.6-12.8)$

$22.9(18.0-27.3)$

$$
2.36(1.6-3.8)
$$

$10.8(9.0-12.3)$

$10.7(8.4-14.1)$
$19.1(15.6-23.5)$
$8.6(4.5-16.7)$
$36.2(19.9-54.9)$
$54.5(47.6-62.4)$
$4.1(3.6-4.7)$
$5.9(4.5-7.3)$

$2.8(2.3-3.4)$

6.7 (5.2-7.7)
Black

$\mathrm{N}=186,373$

$12.4(9.7-15.5)$

$68.8(62.8-75.7)$

$15.8(12.3-20.4)$

$10.7(7.9-13.0)$

$23.6(19.4-28.7)$

$2.46(1.71-3.81)$

$10.3(8.8-11.6)$

$10.7(8.5-14.2)$

$10.8(8.5-14.2)$

$$
\begin{gathered}
19.2(15.6-23.6) \\
7.7(3.8-14.9) \\
28.6(15.2-47.6) \\
54.5(47.6-62.3) \\
4.0(3.5-4.7) \\
5.52(4.3-7.2)
\end{gathered}
$$

$$
\begin{gathered}
19.5(16.1-23.7) \\
9.8(5.6-21.1) \\
50.7(36.24-63.36) \\
54.4(46.7-61.6) \\
4.3(3.7-4.9) \\
6.4(4.97-7.51)
\end{gathered}
$$

2.7 (2.2-3.3)

6.5 (5.0-7.5)
$3.1(2.5-3.6)$

$7.2(6.0-8.7)$
Other

( $\mathrm{N}=65,746)$

12.6 (9.7-16.3)

$69.1(64.0-77.2)$

13.7 (9.7-17.9)

$9.6(7.1-12.7)$

$24.4(18.9-28.9)$

$3.6(2.01-6.54)$

$9.6(8.8-11.5)$

$9.6(7.5-12.3)$

$17.9(14.4-21.8)$

$13.3(6.6-23.3)$

49.7 (28.2-63.9)

57.6 (50.3-68.9)

$4.0(3.5-4.7)$

$6.75(5.0-8.5)$

$3.0(2.4-3.4)$ 6.8 (5.3-7.5)

service-connected disability rating, income, recent military service, and other factors.

Abbreviations: P25 $25^{\text {th }}$ Percentile; P75, $75^{\text {th }}$ Percentile 
Table 2. Adjusted risk ratios $(95 \mathrm{Cl})$ for receiving a positive COVID-19 test result among Veterans Enrolled in Active Care at the Veterans Health Administration (VHA) who obtained a COVID-19 test, February 8-December 28, 2020. ${ }^{\text {a }}$

\begin{tabular}{|c|c|c|c|c|c|c|c|c|c|}
\hline \multirow[b]{2}{*}{$\begin{array}{l}\text { Percentage of } \\
\text { Persons in } \\
\text { Deep Poverty }\end{array}$} & \multirow[b]{2}{*}{$\begin{array}{l}3.5-11.2 \\
>11.2-14.7 \\
>14.7-17.0 \\
>17.0-48.6\end{array}$} & \multicolumn{2}{|c|}{$\begin{array}{c}\text { All } \\
(\mathrm{N}=778,599)\end{array}$} & \multicolumn{2}{|c|}{$\begin{array}{c}\text { White } \\
(\mathrm{N}=526,480)\end{array}$} & \multicolumn{2}{|c|}{$\begin{array}{c}\text { Black } \\
(\mathrm{N}=186,373)\end{array}$} & \multicolumn{2}{|c|}{$\begin{array}{c}\text { Other } \\
(\mathrm{N}=65,746)\end{array}$} \\
\hline & & $\begin{array}{l}\text { REF } \\
0.96 \\
0.94 \\
0.94\end{array}$ & $\begin{array}{c}-- \\
{[0.89,1.02]} \\
{[0.86,1.03]} \\
{[0.83,1.05]}\end{array}$ & $\begin{array}{l}\text { REF } \\
0.97 \\
0.95 \\
0.96\end{array}$ & $\begin{array}{c}-- \\
{[0.90,1.04]} \\
{[0.87,1.05]} \\
{[0.84,1.09]}\end{array}$ & $\begin{array}{l}\text { REF } \\
0.88 \\
0.89 \\
0.87\end{array}$ & $\begin{array}{c}-- \\
{[0.79,0.98]} \\
{[0.78,1.01]} \\
{[0.73,1.03]}\end{array}$ & $\begin{array}{l}\text { REF } \\
0.96 \\
0.89 \\
0.87\end{array}$ & $\begin{array}{c}-- \\
{[0.84,1.10]} \\
{[0.76,1.04]} \\
{[0.72,1.04]}\end{array}$ \\
\hline $\begin{array}{l}\text { Percentage } \\
\text { without 4+ } \\
\text { Years College, } \\
\text { Ages 25+ }\end{array}$ & $\begin{array}{l}21.9-65.1 \\
>65.1-69.9 \\
>69.9-77.8 \\
>77.8-95.1\end{array}$ & $\begin{array}{l}\text { REF } \\
1.14 \\
1.12 \\
1.23\end{array}$ & $\begin{array}{c}-- \\
{[1.08,1.22]} \\
{[1.04,1.21]} \\
{[1.10,1.37]}\end{array}$ & $\begin{array}{l}\text { REF } \\
1.19 \\
1.16 \\
1.24\end{array}$ & $\begin{array}{c}-- \\
{[1.12,1.26]} \\
{[1.07,1.26]} \\
{[1.11,1.38]}\end{array}$ & $\begin{array}{l}\text { REF } \\
1.06 \\
1.02 \\
1.14\end{array}$ & $\begin{array}{c}-- \\
{[0.98,1.14]} \\
{[0.93,1.12]} \\
{[0.99,1.31]}\end{array}$ & $\begin{array}{l}\text { REF } \\
1.08 \\
1.12 \\
1.31\end{array}$ & $\begin{array}{c}-- \\
{[0.95,1.23]} \\
{[0.98,1.28]} \\
{[1.09,1.59]}\end{array}$ \\
\hline $\begin{array}{l}\text { Percentage } \\
\text { Food } \\
\text { Stamps/SNAP } \\
\text { Recipients }\end{array}$ & $\begin{array}{l}0.4-10.8 \\
>10.8-14.6 \\
>14.6-18.3 \\
>18.3-57.3\end{array}$ & $\begin{array}{l}\text { REF } \\
1.01 \\
1.07 \\
1.06\end{array}$ & $\begin{array}{c}-- \\
{[0.94,1.09]} \\
{[0.99,1.16]} \\
{[0.95,1.18]}\end{array}$ & $\begin{array}{l}\text { REF } \\
1.00 \\
1.05 \\
1.06\end{array}$ & $\begin{array}{c}-- \\
{[0.92,1.09]} \\
{[0.96,1.15]} \\
{[0.94,1.21]}\end{array}$ & $\begin{array}{l}\text { REF } \\
1.05 \\
1.10 \\
0.97\end{array}$ & $\begin{array}{c}-- \\
{[0.96,1.15]} \\
{[0.98,1.24]} \\
{[0.83,1.13]}\end{array}$ & $\begin{array}{l}\text { REF } \\
1.06 \\
1.08 \\
1.18\end{array}$ & $\begin{array}{c}-- \\
{[0.96,1.18} \\
{[0.94,1.24} \\
{[0.98,1.43}\end{array}$ \\
\hline $\begin{array}{l}\text { Percentage } \\
\text { Eligible for } \\
\text { Medicaid }\end{array}$ & $\begin{array}{l}0.6-18.0 \\
>18.0-22.9 \\
>22.9-27.3 \\
>27.3-62.0\end{array}$ & $\begin{array}{l}\text { REF } \\
1.04 \\
1.08 \\
1.17\end{array}$ & $\begin{array}{c}-- \\
{[0.97,1.11]} \\
{[0.99,1.22]} \\
{[1.05,1.37]}\end{array}$ & $\begin{array}{l}\text { REF } \\
1.01 \\
1.07 \\
1.16\end{array}$ & $\begin{array}{c}-- \\
{[0.94,1.01]} \\
{[0.97,1.17]} \\
{[1.02,1.32]}\end{array}$ & $\begin{array}{l}\text { REF } \\
1.06 \\
1.10 \\
1.20\end{array}$ & $\begin{array}{c}-- \\
{[0.99,1.14]} \\
{[0.99,1.22]} \\
{[1.05,1.37]}\end{array}$ & $\begin{array}{l}\text { REF } \\
1.12 \\
1.11 \\
1.21\end{array}$ & $\begin{array}{c}-- \\
{[1.00,1.24} \\
{[0.99 .1 .25} \\
{[1.03,1.44]}\end{array}$ \\
\hline $\begin{array}{l}\text { Percentage in } \\
\text { Crowded } \\
\text { Housing }\end{array}$ & $\begin{array}{l}0.0-1.6 \\
>1.6-2.4 \\
>2.4-3.8 \\
>3.8-34.9\end{array}$ & $\begin{array}{l}\text { REF } \\
1.01 \\
1.10 \\
1.07\end{array}$ & $\begin{array}{c}-- \\
{[0.96,1.07]} \\
{[1.04,1.17]} \\
{[0.99,1.17]}\end{array}$ & $\begin{array}{l}\text { REF } \\
0.99 \\
1.11 \\
1.10\end{array}$ & $\begin{array}{c}-- \\
{[0.94,1.06]} \\
{[1.03,1.19]} \\
{[0.99,1.21]}\end{array}$ & $\begin{array}{l}\text { REF } \\
1.04 \\
1.07 \\
0.99\end{array}$ & $\begin{array}{c}-- \\
{[0.98,1.10]} \\
{[1.00,1.15]} \\
{[0.88,1.11]}\end{array}$ & $\begin{array}{l}\text { REF } \\
1.01 \\
1.08 \\
1.12\end{array}$ & $\begin{array}{c}-- \\
{[0.91,1.13} \\
{[0.95,1.21]} \\
{[0.93,1.34]}\end{array}$ \\
\hline $\begin{array}{l}\text { Percentage } \\
\text { Without } \\
\text { Computer }\end{array}$ & $\begin{array}{l}1.4-8.4 \\
>8.4-10.7 \\
>10.7-14.1 \\
>14.1-61.7\end{array}$ & $\begin{array}{l}\text { REF } \\
1.04 \\
1.06 \\
1.05\end{array}$ & $\begin{array}{c}-- \\
{[0.97,1.13]} \\
{[0.96,1.18]} \\
{[0.93,1.19]}\end{array}$ & $\begin{array}{l}\text { REF } \\
1.08 \\
1.09 \\
1.08\end{array}$ & $\begin{array}{c}-- \\
{[0.99,1.18]} \\
{[0.96,1.23]} \\
{[0.94,1.24]}\end{array}$ & $\begin{array}{l}\text { REF } \\
0.98 \\
1.07 \\
1.06\end{array}$ & $\begin{array}{c}-- \\
{[0.93,1.05]} \\
{[0.97,1.17]} \\
{[0.92,1.21]}\end{array}$ & $\begin{array}{l}\text { REF } \\
1.07 \\
1.09 \\
1.09\end{array}$ & $\begin{array}{c}-- \\
{[0.94,1.22]} \\
{[0.90,1.32} \\
{[0.86,1.37]}\end{array}$ \\
\hline $\begin{array}{l}\text { Percentage } \\
\text { Without } \\
\text { Broadband } \\
\text { Internet }\end{array}$ & $\begin{array}{l}6.0-15.6 \\
>15.6-19.1 \\
>19.1-23.5 \\
>23.5-74.3\end{array}$ & $\begin{array}{l}\text { REF } \\
1.04 \\
1.02 \\
1.03\end{array}$ & $\begin{array}{c}-- \\
{[0.97,1.12]} \\
{[0.92,1.13]} \\
{[0.92,1.16]}\end{array}$ & $\begin{array}{l}\text { REF } \\
1.02 \\
0.99 \\
1.01\end{array}$ & $\begin{array}{c}-- \\
{[0.94,1.10]} \\
{[0.88,1.11]} \\
{[0.88,1.14]}\end{array}$ & $\begin{array}{l}\text { REF } \\
1.06 \\
1.07 \\
1.06\end{array}$ & $\begin{array}{c}-- \\
{[0.97,1.15]} \\
{[0.94,1.21]} \\
{[0.93,1.21]}\end{array}$ & $\begin{array}{l}\text { REF } \\
1.05 \\
0.97 \\
0.91\end{array}$ & $\begin{array}{c}-- \\
{[0.91,1.21]} \\
{[0.80,1.17]} \\
{[0.72,1.16]}\end{array}$ \\
\hline
\end{tabular}




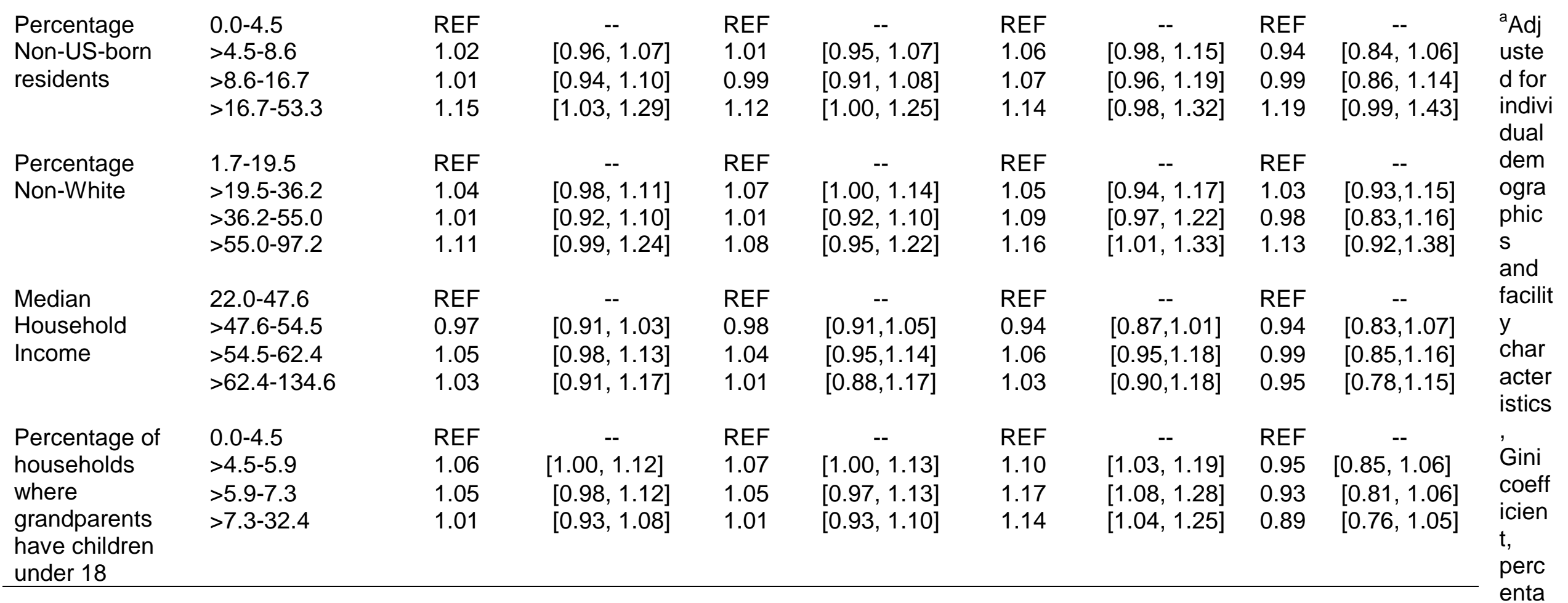

ge aged 65+ living alone, rural/urban/highly rural, unemployment rate (2017), without health insurance. 
Supplementary Table 1. Data Dictionary for county-level socioeconomic measures.

\section{Measure}

Percentage of Persons in Deep Poverty, 2017

Percentage Persons 65+ in Deep Poverty, 2017

Percentage without High School Diploma,

Ages 25+, 2013-2017 (5-year)

Percentage without 4+ Years College, Ages

25+, 2013-2017 (5-year)

Percentage Food Stamp/Supplemental

Nutrition Assistance Program Recipients,

2015

Percentage Living in Poverty, All Ages, 2016

Percentage without Health Insurance, Under Age 65, 2016

Percentage Eligible for Medicaid, All Ages,

2012

Percentage in Crowded Housing, 2013-2017

Percentage 65+ living alone, 2018

Percentage of households where grandparent have children under 18, 2018

Percentage of Households without a computer, 2018

Percentage of households without broadband, 2018

Percentage of US Non-Native Residents, 2018

Percentage Non-White, 2018

Median Household Income (thousands), 2016

Income Inequality (GINI Index), 2018

Unemployment Rate Ages 16+, 2017

\section{Source}

Area Health Resources

Files

Area Health Resources

Files

Centers for Disease

Control and Prevention

Centers for Disease

Control and Prevention

Centers for Disease

Control and Prevention

Centers for Disease

Control and Prevention

Centers for Disease

Control and Prevention

Centers for Disease

Control and Prevention

Diversity Data for Kids

US Census Bureau

US Census Bureau

US Census Bureau

US Census Bureau

US Census Bureau

US Census Bureau

Centers for Disease

Control and Prevention

US Census Bureau

Centers for Disease

Control and Prevention

\section{Source Details}

(.1) Table B17024 American Community

Survey 2013-2017

(.1) Table B17024 American Community

Survey 2013-2017

American Community Survey 2013-2017

American Community Survey 2013-2017

Health Resources and Services

Administration; Area Health Resources File

U.S. Census Bureau (SAIPE); Small Area

Income and Policy Estimates

U.S. Census Bureau (SAHIE)

Small Area Health Insurance Estimates

Health Resources and Services

Administration; Area Health Resources File

Diversity Data Kids

(25014_1_P_050_5_crowded housing_race)

American Community Survey $\overline{5}$-year 2018

Table DP02

American Community Survey 5-year 2018

Table DP02

American Community Survey 5-year 2018

Table DP02

American Community Survey 5-year 2018

Table DP02

American Community Survey 5-year 2018

Table DP02

American Community Survey 5-year 2018

Table DP02

U.S. Census Bureau (SAIPE); Small Area

Income and Policy Estimates

American Community Survey 5-year 2018

U.S. Bureau of Labor Statistics; Local Area Unemployment Statistics

\section{Original Variable}

F15419-13

F15425-13

prop_edu_less_highsch ool

prop_edu_less_college

prop_food_stamp_sp_re

cip

prop_poverty

prop_uninsured

prop_medicaid_eligible

total_est

DP02_0012PE

DP02_0043PE

DP02_0151PE

DP02_0152PE

DP02_0087PE

DP02_0087E

NA

B19083_001E unemployment_rate

${ }^{1}$ Crowded housing defined as the number of occupied housing units with more than one occupant per room divided by the number of occupied housing units, times 100 , for the total population and by race/ethnicity. 